Brit. J. industr. Med., 1960, 17, 25.

\title{
STUDIES ON THE NATURE OF SILICOSIS
}

\section{A SUGGESTED MECHANISM OF FIBROGENESIS}

\author{
BY \\ P. F. HOLT and C. W. WENT \\ From the Department of Chemistry, Reading University
}

(RECEIVED FOR PUBLICATION MARCH 10, 1959)

\begin{abstract}
One theory which seeks to explain the production of fibrous tissue in silicosis postulates the interaction of collagen precursors with polysilicic acid. This stage has been questioned because it is doubtful whether polymerized silicic acid is normally formed when quartz dissolves in aqueous media. A mechanism by which silicic acid may be polymerized in vivo has been demonstrated by studying the properties of polyamide monolayers, which behave like collagen monolayers when spread on silicic acid substrates. The silicic acid which is adsorbed beneath the monolayer polymerizes when the polyamide films are subjected to pressure.

In fibrogenesis, the polysaccharides which are produced in large amounts may serve to prevent the premature agglomeration of the collagen units. Later, when the concentration of the polysaccharide is largely reduced, the polysaccharide may assist in aligning and cementing the units together to form fibres. The physiological process is probably reversible but, if silicic acid takes the role of the polysaccharide in this second stage, the building up of fibres is likely to be an irreversible process.
\end{abstract}

Defects of the solubility theory, which sought to explain the fibrogenesis caused by silica, were examined in earlier publications. An attempt was made to modify the theory and so to remove some of the obvious anomalies (Holt and Osborne, 1953a and b; Holt and Yates, 1954). The original solubility theory (for a review, see Holt, 1957) regarded the silicic acid, produced when a siliceous dust dissolves in the tissue fluids, as a cell poison. However, attempts to demonstrate the toxicity of silicic acid failed and none of the evidence on which the theory was based appears now to be valid. It seems certain that silicic acid in tissue fluids will not of itself initiate fibrosis. Silicic acid appears to be active only if it is liberated in the cytoplasm of a fibroblast.

The modified theory suggested that simple silicic acids were formed in the cytoplasm of the dust cells and that this, diffusing from the cells, reacted with collagen precursors in the same manner as mucopolysaccharides are believed to react in the formation of normal fibres, producing stabilized collagen. Since these ideas were published, certain investigations of a fundamental nature have been made, the results of which are relevant to this discussion. A part of this material, as a contribution to knowledge in pure chemistry, has already appeared (Holt and
Bowcott, 1954; Clark, Holt, and Went, 1957; Clark and Holt, 1957). The evidence for the interaction of proteins with silicic acid was described and the difference between the behaviour of collagen and that of other proteins was emphasized. Other experimental results, more directly related to the problem of silicosis, are recorded here.

Silicosis is essentially a disease in which collagen, a normal constituent, is formed in the lung in abnormal quantities. When silica particles enter -the lung they are taken up by monocytes which become fibroblasts. Collagen is synthesized by the fibroblast, in the first place probably as ultramicroscopic rods or even spheres of protein which pass through the cell wall and, outside the cell, coalesce to form collagen fibres. The process of fibre growth is, then, analogous to crystal formation.

Collagen is always associated with polysaccharides and their function is a subject of speculation. Originally the polysaccharides were thought to act as templates on which the protein fibres were built (Partridge, 1948). Certainly chondroitin sulphate, for example, can precipitate small fibres from a solution of collagen (Cohen, 1942). Collagen fibres can be precipitated by simpler means, for example, by changing the tonicity of a saline solution of collagen (Nageotte and Guyon, 1935). Later, it was 
proved experimentally that polysaccharides will stabilize collagen fibres, retarding changes which normally occur on heating (Jackson, 1953, 1954). More recently (Holt and Went, 1956), the polysaccharide secreted with the collagen precursor was thought to prevent premature coalescence of the protein fibrils, the large molecules preventing the protein chains from aligning one with another and so retarding the crystallization of the collagen. A mechanism by which the hydroxyl groups of water, and presumably those of polysaccharides also, may be attached to the collagen surface has been described (Fraser and Macrae, 1959).

It is conceivable that the polysaccharides serve all these functions. The very large amounts which are secreted with the collagen could hardly fail to hinder the development of the fibres in the first place but the amount of the polysaccharides later decreases and it may then assist in the alignment of the fibrils and stabilize the fibres when formed.

It is becoming apparent that the normal pattern of many tissues always involves both protein and polysaccharides. The permeability of the tissue depends on the amount and the degree of polymerization of the polysaccharides; the permeability is increased by the degradation or removal of the polysaccharides by polysaccharases. Even the control of the water content of the organism by the kidney appears to involve this system (Ginetzinsky, 1958). The polysaccharide, hyaluronic acid, is reported to be the substance controlling the permeability of the tubules and the permeability is largely increased by hyaluronidase. A polysaccharide-polysaccharase system of control for the production of collagen fibres would not, then, constitute a novel system.

The following sequence might be involved in fibrogenesis when silica is absent. The response of the fibroblast to the stimulus is the production of the elementary units of the protein collagen, together with large amounts of polysaccharide. The polysaccharide prevents the immediate aggregation of the protein units and the protein-carbohydrate complex moves away from the cell. The polysaccharide is gradually removed by polysaccharases and eventually the fibrils, with a layer of adsorbed polysaccharide, align to form fibres. The polysaccharide then acts as a cement substance between the fibrils but its amount is steadily reduced until only a minimal amount-perhaps a monolayer-remains. This view of the formation of collagen fibres is in keeping with the observations, made by electron micrography, that the carbohydrate associated with reticulin fibres is on the surface but that it is more closely associated with the older, fully-developed collagen fibres (Schwarz, 1957).
When silicic acid polymerizes a macromolecular structure is produced which can combine with and stabilize collagen (Clark, 1955). The possibility was considered that polysilicic acid behaves like the polysaccharides in the process of fibrogenesis as it is found in silicosis. Formed by the dissolution of silica particles in the cytoplasm, silicic acid would not be produced in a concentration likely to retard the production of fibres, but polysaccharides are simultaneously formed in large amount (Curran, 1952, 1953) and may serve this purpose. Polysilicic acid might serve as a template, and it will certainly stabilize collagen fibres even more efficiently than polysaccharides. It is not, of course, removed by polysaccharases.

This explanation of fibrogenesis assumes the polymerization of the silicic acid which is produced by the dissolution of silica particles; but silica dissolves slowly in aqueous media and produces a very dilute solution. Holt and Yates (1954) suggested that, because of the very high concentration of fine particles and consequent large surface of silica in the aggregation of monocytes, sufficient silicic acid might be formed for polymerization to occur. However, Wheatley (1957) found no polysilicic acid in extracts of silica, although Sakabe, Hasegawa, and Yamada (1956) reported that polysilicic acid is so formed. Their observations were all made on the simple silica-water system.

Some biological systems are certainly capable of concentrating extremely dilute solutions of orthosilicic acid and from them can produce silica itself. It happens in some plants, which produce silica in their leaves. Diatoms (Asterionella) build silica structures from very dilute solutions and the amount of silica in the structure is independent of the concentration of silicic acid in the environment (Einsele and Grim, 1938) which may be as low as $0.2 \mathrm{mg}$. per litre (Lund, 1950). Geological flints, which are often the inside casts of sea organisms, show that a mechanism exists by which even the trace of silicic acid in sea water can be concentrated to produce large masses of silica. In these cases the production of silica must entail the concentration and polymerization of orthosilicic acid in very dilute solutions, but by a process about which nothing is known and which has not been imitated in vitro.

Studies on the interaction of silicic acid with polyamide films and with proteins suggest that the concentration and polymerization of silicic acid could be effected by the collagen itself. The technique (Holt and Went, 1956) by which the studies are made consists in spreading a monomolecular layer of the polymer on saline and on a dilute solution of silicic acid. The films are slowly compressed. A graph drawn to illustrate the relation between the 
applied pressure and the area of the film has a shape which is characteristic of the type of material. The shape of the curve may be altered if the silicic acid in the substrate interacts with the protein. The conditions under which silicic acid interacts with various proteins have been described (Holt and Bowcott, 1954; Clark et al., 1957; Holt and Went, 1956).

Polyamides make excellent simplified protein models. They possess a sequence of peptide links separated by hydrocarbon chains and provide a structure similar to a protein backbone without the complication given by side chains.

The structures may be compared thus:

Polyamide ...

Protein ...

$$
-\mathrm{NHCO}-\left(\mathrm{CH}_{2}\right)_{n}-\mathrm{CONH}-\left(\mathrm{CH}_{2}\right)_{n}-
$$

$\mathbf{R}=$ amino-acid residue.

$$
\text { - } \mathrm{CONH}-\mathrm{CHR}-\mathrm{CONH}-\mathrm{CHR}-
$$

(Alternate peptide links are reversed in most polyamides.)

They are readily available and solutions of suitable concentration can be made much more easily than from collagen. Films of polyamides give force/area curves which are shallow, resembling those of collagen rather than those of most other proteins. In this study, polyamide films were used, the work previously published having shown the similarity in behaviour between polyamides and collagen films.

\section{Experimental}

Polyamides.-Solutions containing $0.01 \%$ of an amorphous copolymer, 66/6, 10/6 were spread from an ethanol + benzyl alcohol $(3: 1)$ solution.

Substrates.-To obviate complications due to polyvalent anions, substrates were not buffered. The $p \mathrm{H}$ was adjusted ( $\mathrm{HCl}$ ) before, and accurately measured after, each experiment. Polyamides were spread on $0.5 \mathrm{M} \mathrm{NaCl}$.

Sodium silicate solutions $(0.1 \mathrm{M})$ were either prepared by fusing pure vitreous silica (Thermal Syndicate Limited) with sodium carbonate (1:4) and dissolving the melt in water, or from sodium metasilicate. Solutions were treated with activated charcoal for 12 hours and filtered.

Silicic acid was prepared by adding $2 \mathrm{~N} \mathrm{HCl}$ to the silicate solution. Polymerized silicic acid solutions, prepared by allowing $0.08 \mathrm{M}$ silicic acid at $p \mathrm{H} 6.0$ to stand up for up to $40 \mathrm{~min}$. at $25-30^{\circ}$, were stabilized by acidifying with $2 \mathrm{~N} \mathrm{HCl}$ to $p \mathrm{H} 1.5$ to 2.0 and diluting to $0.05 \mathrm{M}$.

In solutions more dilute than $0.002 \mathrm{M}$ orthosilicic acid does not polymerize. At higher concentrations at equilibrium, $0.002 \mathrm{M}$ orthosilicic acid is always present and the excess is polymerized. Depolymerization of diluted polysilicic acid sols was found to be slow at $25^{\circ}$; below $p \mathrm{H} 6$ little additional monomer was formed in, say, 10 minutes. The distribution of polymer sizes was, then, reasonably constant in all the prepolymerized substrates; they contained larger polymers and a range of smaller polymers down to orthosilicic acid.

Procedure.-Measurements of surface pressure were made with a Langmuir-Adam balance, using a waxed perspex trough $(12.6 \times 72 \mathrm{~cm}$.). The polyamide was spread from solution by placing small drops from a glass micrometer syringe on the surface of the substrate, normally on an area of $2.2 \mathrm{~m} .2 / \mathrm{mg}$. of polyamide.

Qualitative information about the viscosity was obtained by dusting films with talc and blowing on the surface.

\section{Results and Discussion}

When a polyamide film is formed on an aqueous salt solution and is then compressed, the relevant force/area curve is shallow (Fig. 1, curve A). If the sub-solution contains polysilicic acid in a concentra-

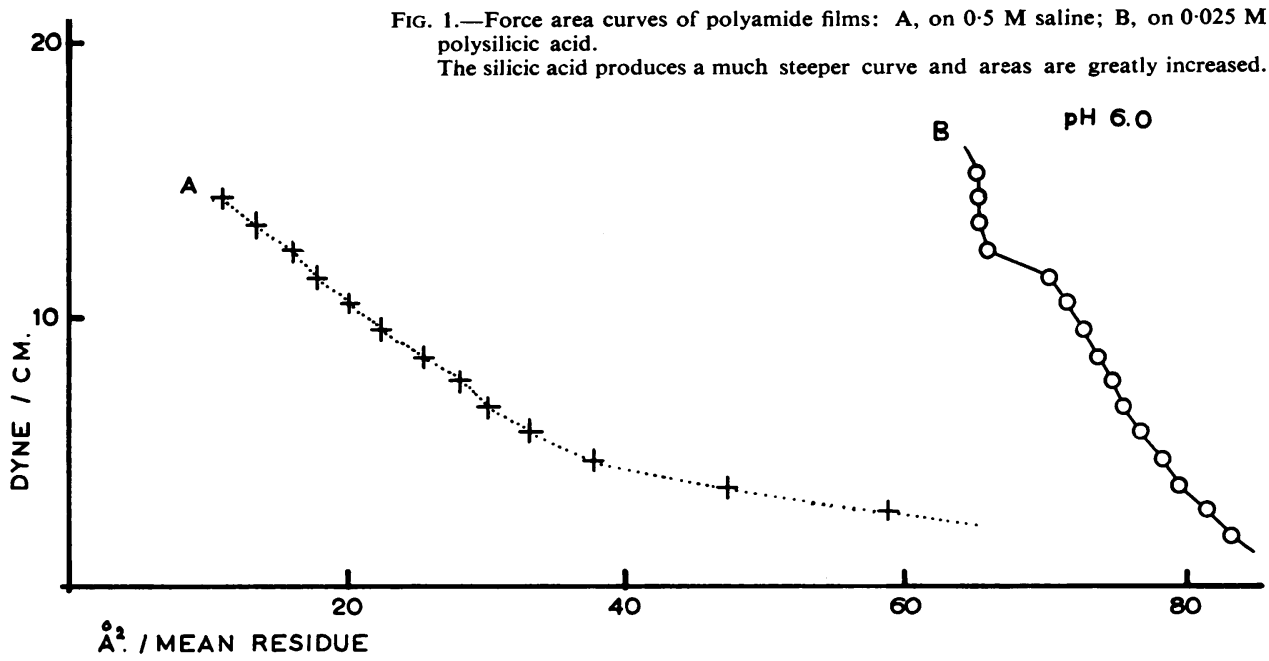




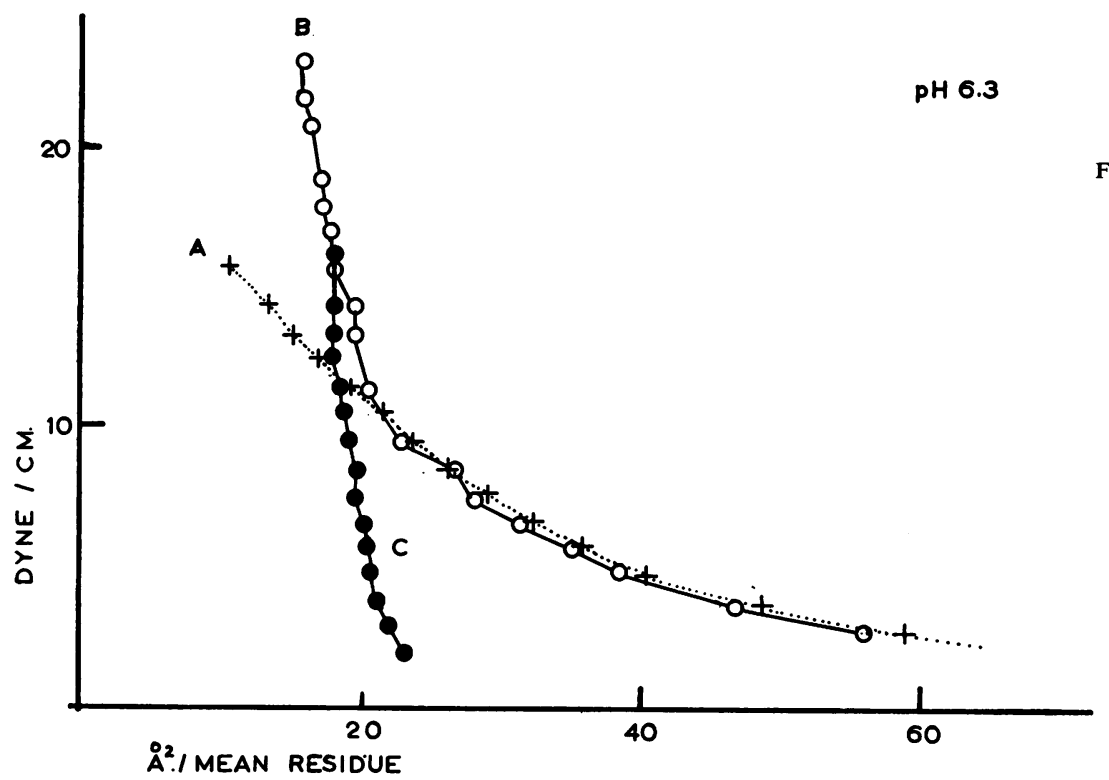

Fig. 2.-Force area curves of polyamide films: A, on $0.5 \mathrm{M}$ saline; $B$, on $0.5 \mathrm{M}$ saline $\times$ $0.0001 \mathrm{M}$ silicic acid; C, same as $B$, second compression. The silicic acid is too dilute to affect the film until a pressure of 10 dyne $/ \mathrm{cm}$. is applied. The curve then rises steeply. Curve $\mathrm{C}$ shows that the protein-silicic acid complex is stable.

tion of say, $0.025 \mathrm{M}$, the form of the curve is altered. It rises steeply, and the change indicates that interaction has occurred and the film has been stabilized. Under these conditions the polysilicic acid particles which are adsorbed to the underside of the film approach one another so closely that interaction can occur between them, there is two-dimensional polymerization, and a permanent network forms. The film is said to be "tanned".

When the polyamide is spread on a solution of polysilicic acid diluted to $0.0001 \mathrm{M}$, the silicic acid apparently has no effect on the properties of the film at lower pressures, but above a force of about 10 dyne/cm., the curve rises steeply away from the

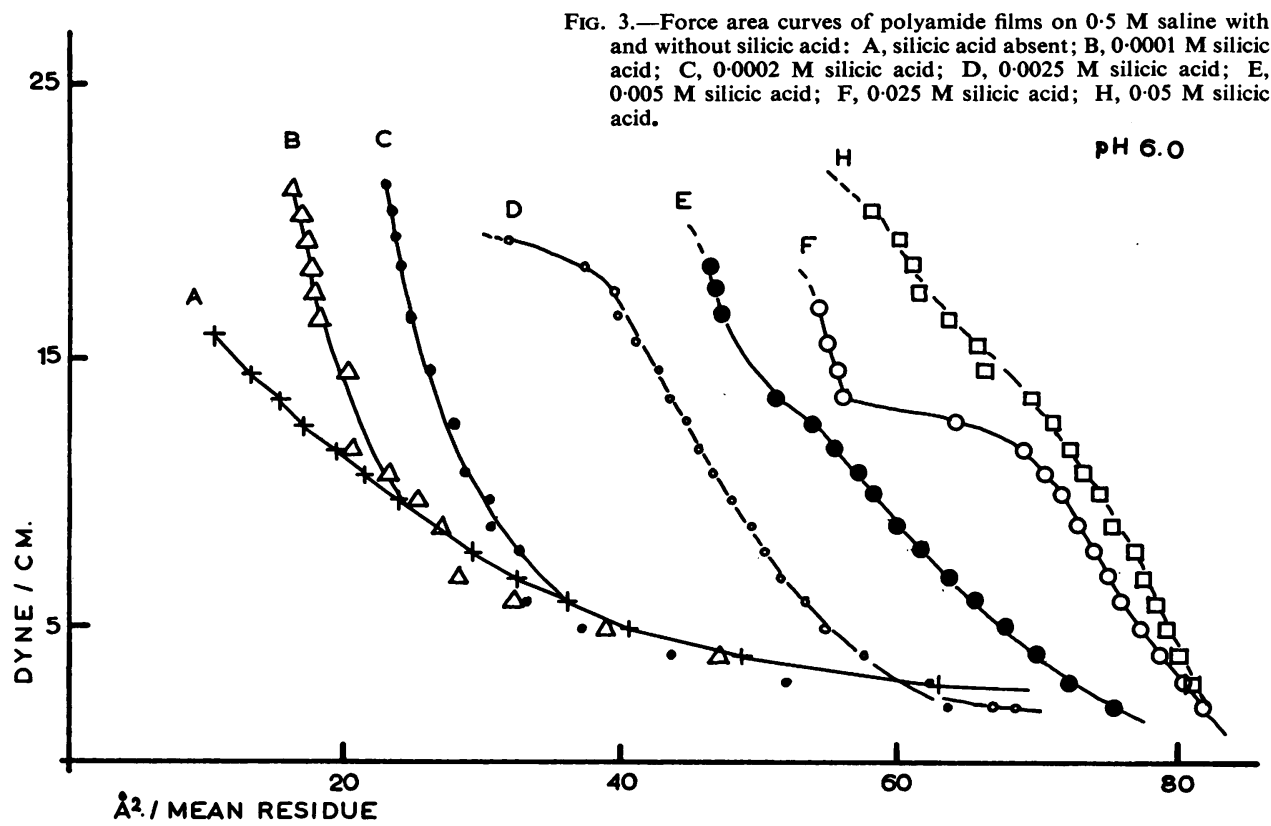


normal (Fig. 2, curve B). Whereas in the absence of silicic acid the curves show that the films break down at a pressure of about 15 dyne/cm. (Fig. 3, curve A), the films are now stable beyond 25 dyne $/ \mathrm{cm}$. Moreover, the change on compression, which in the absence of silicic acid is reversible, is no longer so. If the pressure is released and the film is then compressed a second time, there is no large increase in pressure until the area is reduced almost to $20 \mathrm{~A}^{2} /$ mean residue (Fig. 2, curve $C$ ).

These curves show that, although the silicic acid at this very low concentration interacts with the polyamide film, the properties of the film are unaltered until a critical pressure is reached. Then the adsorbed silicic acid units are pushed together close enough for chemical interaction (condensation or hydrogen bonding) to occur between them, and the film becomes coated by a continuous layer of polysilicic acid which need be no more than one unit thick. This is apparent from Fig. 3, where it is seen that the area at which the tanning occurs depends on the concentration of the silicic acid used.

In the experiments described, the films were compressed a few minutes after they had been spread. In other experiments, illustrated in Fig. 4, films were left on the silicic acid substrate for 48 or 60 hours before they were compressed. While the effect of tanning is not observed on solutions of polysilicic acid as dilute as $0.0001 \mathrm{M}$ until the area of the polyamide film is reduced to about $25 \mathrm{~A}^{2} /$ mean residue, it is observed over practically the whole range if the silicic acid is allowed to interact for a long period. This is not due to the silicic acid polymerizing in the substrate, because at this concentration silicic acid does not polymerize at all; on the contrary, it slowly depolymerizes. Even at a concentration of $0.005 \mathrm{M}$, it does not polymerize appreciably (Fig. 5). The silicic acid is slowly and irreversibly adsorbed to the protein layer, the twodimensional concentration increasing until polymerization occurs beneath the film. The polyamide film thus acts as a collecting surface for the silicic acid. In vivo collagen may behave in the same way.

Here, then, is a mechanism by which a polysilicic acid layer may be built up from very dilute solutions of silicic acid and probably even from orthosilicic acid. It provides a feasible mechanism for the stabilization of collagen fibres in silicotıc tissue. Whilst in the environment in which fibrosis is induced collagen may be the adsorbing phase, other suitable macromolecules might be equally effective in other biological systems.

The postulated mechanism of fibrogenesis is, then, that normally the response of the cell to certain

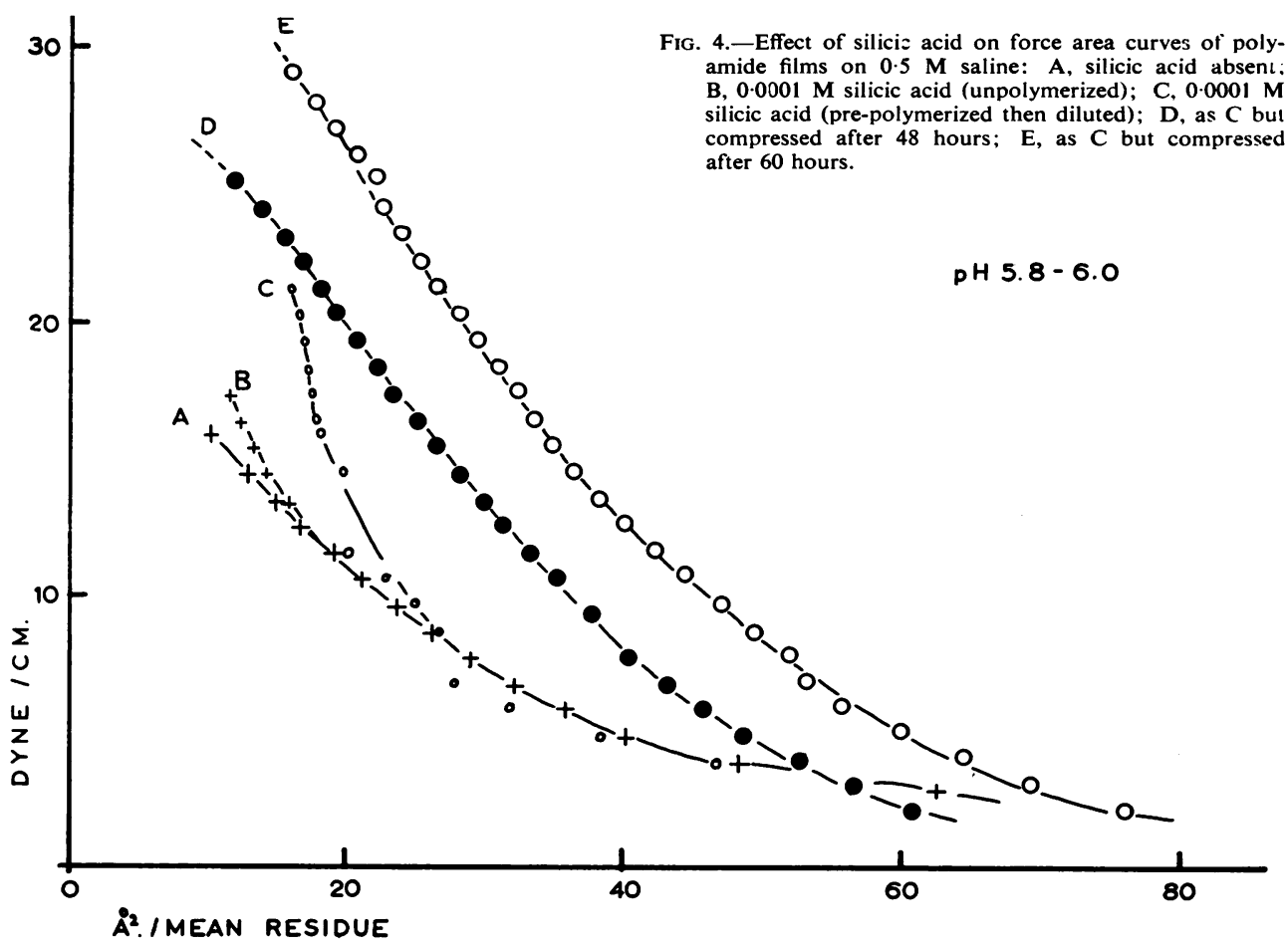




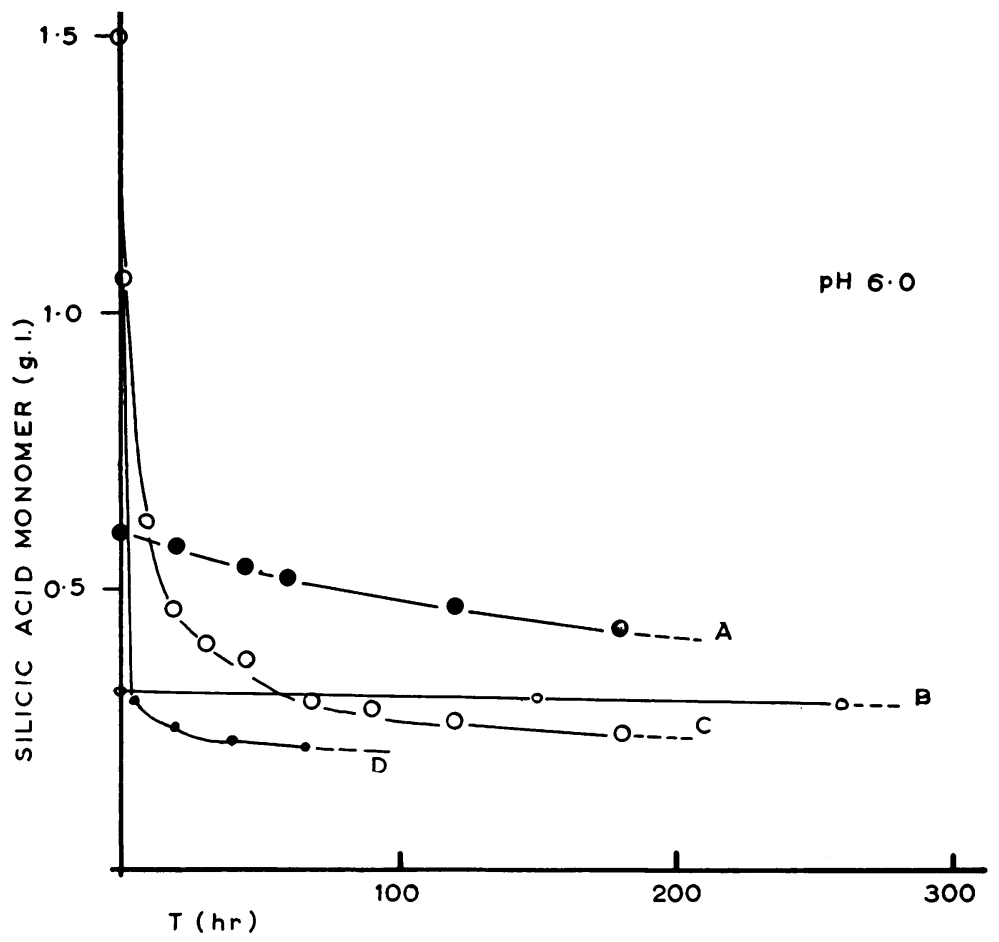

FiG. 5.-Rate of polymerization of silicic acid: variation with concentration. Polymerization is shown by a decrease in the concentration of monomer, measured as molybdatereactive silicic acid. At $0.005 \mathrm{M}$ no polymerization is apparent. Concentrations: A, 0.01 M; B, 0.005 M, C, $0.05 \mathrm{M} ; \mathrm{D}, 0.1 \mathrm{M}$.

stimuli is the production of a collagen precursor in the form of very small units which are prevented from aggregating by the large amounts of polysaccharide which are secreted simultaneously. Most of the polysaccharide is later removed by polysaccharases, allowing the alignment of fibrils and the production of fibres with which a small amount of polysaccharide is associated as a cement substance. In normal fibrogenesis an equilibrium may be established, fibres being built up and others being simultaneously dispersed. When silicic acid is produced along with the collagen precursors, it is adsorbed irreversibly. Aggregation of the collagen chains is prevented by the polysaccharide which is produced by the cells at the same time, but once the complex has diffused away from the cells and the polysaccharide is hydrolised, the adsorbed silicic acid provides a cement substance which is permanent; there is no dispersion of the fibres.

This postulated mechanism is presented as a guide to future investigations. Considerable effort has been directed in some laboratories, following the introduction of the first extended solubility theory, towards the demonstration of the presence or absence of polysilicic acid in silicotic tissue. It must be apparent that there can be no justification for the expectation that silica gel or high polymers of the usual type occur in the tissues. The amount of silica available is too small, and if large quantities of high polymers were present, they could only retard fibrogenesis. The polymerization may only occur in two dimensions, along the protein fibres.

The authors are grateful to the British Steel Castings Research Association and to the British Rayon Research Association for financial assistance.

\section{REFERENCES}

Clark, S. G. (1955). Ph.D. Thesis, University of Reading.

- , and Holt, P. F. (1957). Trans. Faraday Soc., 53, 1509.

$\longrightarrow,-$, and Went, C. W. (1957). Ibid., 53, 1500.

Cohen, S. S. (1942). J. biol. Chem., 144, 353.

Curran, R. C. (1952). Brit. J. exp. Path., 33, 82

- (1953). J. Path. Bact., 66, 271.

Einsele, W., and Grim., J. (1938). Z. Bot., 32, 545.

Fraser, R. D. B., and Macrae, T. P. (1959). Nature (Lond.), 183, 179.

Ginetzinsky, A. G. (1958). Ibid., 182, 1218.

Holt, P. F. (1957). Pneumoconiosis. Arnold, London.

, and Bowcott, J. E. L. (1954). Biochem. J., 57, 471

, and Osborne, S. G. (1953a). Nature (Lond.), 171, 892.

- , (1953b). Brit. J. industr. Med., 10, 152 .

-, and Went, C. W. (1956). In Symposium on Techniques in Polymer Science. Royal Institute of Chemistry. Lectures, Monographs and Reports 1956, No. 5. London.

- , and Yates, D. M. (1954). Brit. J. exp. Path., 35, 52.

Jackson, D. S. (1953). Biochem. J., 54, 638. (1954). Ibid., 56, 699.

Lund, J. W. G. (1950). J. Ecol., 38, 1.

Nageotte, J., and Guyon, L. (1935). Arch. Sci. biol. (Napoli), 41, 1. Partridge, S. M. (1948). Biochem. J., 43, 387.

Partridge, S. M. (1948), Biochem. J., 43, 387. 1956). Bull. Inst. publ. Sakabe, H., Hasegawa, T., and Yan.
Hth. (Tokyo), 5, No. 2, p. 7.

Schwarz, W. (1957). In Connective Tissue, A Symposium organized by the Council for International Organizations of Medical Sciences, 1956, p. 144. Ed. R. E. Tunbridge. Blackwell Scientific Publications, Oxford.

Wheatley, K. (1957). Research, 10, 76. 